\title{
Pyrosequencing and Taxonomic Composition of the Fungal Community from Soil of Tricholoma matsutake in Gyeongju
}

\author{
Minji Jeong ${ }^{1}$, Doo-Ho Choi ${ }^{1}$, Woo-Jae Cheon ${ }^{2}$, and Jong-Guk Kim ${ }^{1 *}$ \\ 'Department of Life Sciences and Biotechnology, Kyungpook National University, Daegu 41566, Republic of Korea \\ ${ }^{2}$ Department of Forest Environment, Gyeongsangbuk-do Forest Environment Research Institute, Gyeong-ju 38174, \\ Republic of Korea
}

Tricholoma matsutake is an ectomycorrhizal fungus that has a symbiotic relationship with the root of Pinus densiflora. Soil microbial communities greatly affect the growth of $T$. matsutake, however, few studies have examined the characteristics of these communities. In the present study, we analyzed soil fungal communities from Gyeongju and Yeongdeok using metagenomic pyrosequencing to investigate differences in fungal species diversity, richness, and taxonomic composition between the soil under T. matsutake fruiting bodies (Sample 2) and soil where the fairy ring of T. matsutake was no longer present (Sample 1). The same spot was investigated three times at intervals of four months to observe changes in the community. In the samples from Yeongdeok, the number of valid reads was lower than that at Gyeongju. The operational taxonomic units of most Sample 2 groups were less than those of Sample 1 groups, indicating that fungal diversity was low in the T. matsutakedominant soil. The soil under the T. matsutake fruiting bodies was dominated by more than $51 \%$ T. matsutake. From fall to the following spring, the ratio of $T$. matsutake decreased. Basidiomycota was the dominant phylum in most samples. G-F1-2, G-F2-2, and Y-F1-2 had the genera Tricholoma, Umbelopsis, Oidiodendron, Sagenomella, Cladophialophora, and Phialocephala in common. G-F1-1, G-F2-1, and Y-F1-1 had 10 genera including Umbelopsis and Sagenomella in common. From fall to the following spring, the amount of phyla Basidiomycota and Mucoromycota gradually decreased but that of phylum Ascomycota increased. We suggest that the genus Umbelopsis is positively related to T. matsutake.

Keywords: Fungal community, Tricholoma matsutake, metagenomics

Received: March 12, 2021 Accepted: March 24, 2021

First published online: March 26, 2021

* Corresponding author Phone: +82-53-950-5379 Fax: +82-53-955-5379

E-mail: kimjg@knu.ac.kr

pISSN 1017-7825 eISSN 1738-8872

Copyright(C) 2021 by The Korean Society for Microbiology and Biotechnology

\section{Introduction}

Tricholoma matsutake, known as the pine mushroom, is an ectomycorrhizal fungus that has a symbiotic relationship with the root of Pinus densiflora trees. T. matsutake is used medicinally and is also one of the most famous food items in Northeast Asia because of its unique flavor and taste [1-6]. In South Korea, the yield of T. matsutake is changeable because environmental factors such as temperature and precipitation greatly affect its growth [7]. For this reason, studies have examined the possibility of creating a stable supply of the pine mushroom, but currently, artificial cultivation is not feasible. The effect of environmental factors (climate, soil condition, microbial community, and others) on its growth and interactions between T. matsutake and P. densiflora requires further research [8-10]. Soil microbial communities, in particular, are important to the mycelial growth of T. matsutake, however, few studies on the characteristics of the communities have been conducted [11-14].

Mycorrhizal fungi are an important group of fungi in the soil ecosystem. They have a mutualistic relationship with living plants, exchanging nutrients and some important compounds [15-19]. The major group of mycorrhizal fungi is the ectomycorrhizae (ECM) fungi group. ECM fungi, including T. matsutake, considerably affect the growth of living plants by colonizing close by the rootlets of living plants. ECM fungi form ECM with roots of host plants and have advantageous symbiotic relationships with them. ECM are symbiotic organs between soil and roots and help the trees absorb water and nutrients, receiving carbohydrates from them in exchange to grow mycelium [20-22]. The fungus Arthrinium phaeospermum promotes mycorrhiza formation, similar to the effect of mycorrhization helper bacteria [23]. T. matsutake is an ectomycorrhizal symbiont that is important to the growth of host plant seedlings [24]. In addition, some bacteria and fungi have positive effects on T. matsutake [25]. The soil fungal community is positively influenced by plant species richness and diversity [26]. The interaction between plants and mycorrhizal fungi plays a key role in the circulation of nutrients and the balance of the ecosystem [27-30]. Therefore, the study of soil fungal communities near T. matsutake should be conducted constantly and persistently. 
Next-generation sequencing (NGS) enables the rapid analysis of massive amounts of DNA sequences. It also permits the analysis of DNA sequences from microorganisms that are hard to cultivate [31-34]. Pyrosequencing is one of the sequencing approaches for NGS and has facilitated the study of mass and diverse microorganisms from soil samples. For this study, we used the Illumina MiSeq sequencing platform, which uses the sequences of ribosomal DNA as DNA barcodes to classify the soil fungi rapidly.

Here, we statistically analyzed the characteristics of soil fungal communities from two regions using metagenomic pyrosequencing with barcode to investigate diversity, species richness, and relative abundance or taxonomic composition of the communities in areas where T. matsutake fruiting bodies are present [35].

\section{Materials and Methods \\ Collection of Soil Samples}

We collected two soil samples each in pine forests in October 2017 in Gyeongju and in November 2017 in Yeongdeok, South Korea. Two samples were also collected in October 2018 and in February and June 2019 at Gyeongju. Immediately after harvesting the fruiting body of T. matsutake, we took soil samples of $10 \sim 15 \mathrm{~cm}$ depth and $2 \mathrm{~cm}$ in diameter from right beneath the fruiting body using a sterilized soil sampler. These samples were called G-F1-2 (2017, Gyeongju), G-F2-2 (2018, Gyeongju), and Y-F1-2 (2017, Yeongdeok) (Table 1). About $25 \sim 30 \mathrm{~cm}$ from the fairy ring, we once again collected soil samples of $10 \sim 15 \mathrm{~cm}$ depth and $2 \mathrm{~cm}$ in diameter. These samples were called G-F1-1 (2017, Gyeongju), G-F2-1 (2018, Gyeongju), and Y-F1-1 (2017, Yeongdeok). The same spots sampled in the fall of 2018 were re-sampled four months later, in February 2019, and these samples were named G-W-2 and G-W-1. They were re-sampled again four months later, in June 2019, and these samples were named G-S-2 and G-S-1. So, '-1' was attached to the end of the name for the samples expected to be T. matsutake-minor (Sample-1) and '-2' was attached to the samples expected to be T. matsutake-dominant (Sample-2).

GPS coordinates of the sampling sites were as follows:

- G-F1: $35^{\circ} 47^{\prime} 22.1^{\prime \prime} \mathrm{N}, 129^{\circ} 14^{\prime} 06.9^{\prime \prime} \mathrm{E}$

- Y-F1: $36^{\circ} 28^{\prime} 39.1^{\prime \prime} \mathrm{N}, 129^{\circ} 22^{\prime} 12.0^{\prime \prime} \mathrm{E}$

- G-F2, G-W, G-S: $35^{\circ} 47^{\prime} 20.0^{\prime \prime} \mathrm{N}, 129^{\circ} 14^{\prime} 09.0^{\prime \prime} \mathrm{E}$

\section{DNA Extraction and Pyrosequencing}

Each $10 \mathrm{~g}$ of soil samples was used for isolation of metagenomic DNA using the FastDNA SPIN Kit for Soil (MP Biomedicals, USA) to conduct the pyrosequencing. Polymerase Chain Reaction (PCR) targeting the ITS2 region was used to amplify isolated chromosomal DNA. The sequences of the forward primer were $5^{\prime}$ AATGATACGGCGACCACCGAGATCTACAC-XXXXXXXX-TCGTCGGCAGCGTC-AGATGTGTATAAGAGACAGGCATCGATGAAGAACGCAGC-3'. The sequences of the reverse primer were 5' - CAAGCAGAAGACGGC ATACGAGAT-XXXXXXXX-GTCTCGTGGGCTCGG-AGATGTGTATAAGAGACAGTCC-TCCGCTTATTGA TATGC-3'. "X" indicates that the barcode sequence differed between samples. A PTC-200 Peltier Thermal Cycler (M) Research, USA) was used to conduct the PCR. For separation of PCR products, identical amounts of PCR products from each sample were used by agarose gel electrophoresis. The products of PCR were purified using the CleanPCR (CleanNA, Netherlands) and the quantification of the purified PCR products was performed with a Quant-iT PicoGreen dsDNA Assay Kit (Invitrogen, USA). PCR products longer than $300 \mathrm{bp}$ were purified, and an Agilent 2100 Bioanalyzer (Agilent Technologies, USA) was used to analyze the base sequence of the DNA fragments [36]. In addition, the Illumina MiSeq sequencing platform (Illumina, USA) was used for the pyrosequencing analysis conducted by Chunlab Inc. (Korea), following the instructions supplied by the manufacturer [37]. Pyrosequencing reads data were submitted to the EMBL-EMI database (www.ebi.ac.uk) under accession number PRJEB42318 (primary) and ERP126157 (secondary).

\section{Statistical Data Analysis and Taxonomic Identification}

The pyrosequencing raw reads were processed by using the barcode sequences. The fusion primers with barcode and low-quality reads were trimmed and removed using Trimmomatic 0.32 [38]. We used CLcommunity software (Chunlab Inc.) for statistical analysis. To identify operational taxonomic units (OTUs) at $97 \%$ sequence similarity [39], the CD-HIT program was used. The Mothur platform [40, 41] was used to calculate the rarefaction curves and diversity indices. For taxonomic composition and relative abundance, random sample subsets were generated with the lowest number of reads. Conditionally, T. matsutake was excluded for analysis of relative abundance and taxonomic composition.

Table 1. Sampling information of ten samples of the soil fungal community related to T. matsutake.

\begin{tabular}{lccccc}
\hline & \multicolumn{2}{c}{ Gyeongju } & \multicolumn{2}{c}{ Yeongdeok } \\
\cline { 2 - 5 } \cline { 4 - 6 } & October & October & February & June & November \\
& 2017 & 2018 & 2019 & 2019 & 2017 \\
\hline 25 30 cm far from the fairy ring & G-F1-1 & G-F2-1 & G-W-1 & G-S-1 & Y-F1-1 \\
Under the fruiting body of T. matsutake & G-F1-2 & G-F2-2 & G-W-2 & G-S-2 & Y-F1-2 \\
\hline
\end{tabular}


Table 2. Pyrosequencing results and statistical analysis of the soil fungal community related to T. matsutake.

\begin{tabular}{lcccccccccc}
\hline & G-F1-1 & G-F1-2 & G-F2-1 & G-F2-2 & G-W-1 & G-W-2 & G-S-1 & G-S-2 & Y-F1-1 & Y-F1-2 \\
\hline Number of Valid reads & 101347 & 94695 & 92141 & 106048 & 56395 & 57412 & 94346 & 102565 & 89952 & 86224 \\
OTUs $^{a}$ & 347 & 203 & 578 & 211 & 554 & 230 & 439 & 469 & 664 & 124 \\
Chao1 $^{\text {b }}$ & 348.68 & 204.38 & 596.83 & 217.52 & 561.17 & 238.12 & 447.52 & 475.43 & 665.60 & 124.58 \\
Shannon $^{c}$ & 2.90 & 2.05 & 3.69 & 0.97 & 3.54 & 1.25 & 2.61 & 2.65 & 4.01 & 1.70 \\
Simpson $^{\mathrm{d}}$ & 0.11 & 0.30 & 0.06 & 0.64 & 0.08 & 0.58 & 0.19 & 0.28 & 0.04 & 0.29 \\
Goods lib. Coverage $^{\mathrm{e}}(\%)$ & 99.98 & 99.99 & 99.92 & 99.98 & 99.92 & 99.94 & 99.96 & 99.96 & 99.98 & 99.99 \\
\hline
\end{tabular}

aTUs: Operational Taxonomic Units

${ }^{\mathrm{b}}$ Chao1: Chao1 estimation for species richness

'Shannon: Shannon index for species diversity, $>0$, higher, more diverse

${ }^{\mathrm{d}}$ Simpson: Simpson index for species diversity, $0 \sim 1,1=$ the simplest

${ }^{\mathrm{e}}$ Goods Lib. Coverage: [1 - (number of singleton OTUs / number of total reads)] × 100

\section{Results and Discussion}

Pyrosequencing Results and Statistical Data Analysis

For fungal communities, the total number of valid reads after preprocessing was 881,125 from all eight samples (Table 2). The number of valid reads of G-F2-2 was greatest, at 106,048, followed by G-S-2 at 102,565, G-F1-1 at 101,347, G-F1-2 at 94,695 , G-S-1 at 94,346, G-F2-1 at 92,141, Y-F1-1 at 89,952, Y-F1-2 at 86,224, G-W-2 at 57,412, and G-W-1 at 56,395. Overall, there were a large enough number of reads to analyze the microbial communities. Obviously, with fewer reads in the winter samples, the overall number of surviving fungi was reduced. However, the spring samples showed that the number of reads increased again and the ecosystem of soil microbes recovered. In the sample from Yeongdeok, the number of valid reads was slightly lower than that from Gyeongju. The OTUs of Y-F1-1 were the highest, at 664, and those of Y-F1-2 were the lowest, at 124. These two samples showed the biggest difference, at 540. On the other hand, the G-S-1 and G-S-2 samples were 439 and 469, respectively, showing 30 different results. The OTUs of most Sample 2 groups were lower than those of Sample 1 groups. Naturally, the Shannon index, which is one of the most important diversity indices, was similar to the number of OTUs. For the most part, the number for Sample 2's was lower. In particular, G-F2-1 and G-F2-2 showed a significantly large difference. The Shannon index measures species richness and evenness, which means that the higher the number, the greater the diversity of the community. Therefore, fungal diversity was low in the soil samples in which T. matsutake was dominant. These results are also shown in the Simpson index results, which are presented together to produce more accurate results. Furthermore, the rarefaction curves supported the results above (Fig. 1). In the rarefaction curves, the number of OTUs increased with the number of reads, and the species richness was shown. This is consistent with previous studies $[12,13,25]$ showing that fungal diversity is low in T. matsutake-dominant soil samples. This confirms that the mycelia of T. matsutake repress the growth of other fungi and are dominant in the soil fungal community.

Comparison of Fungal Communities: Composition of T. matsutake

First, for analysis of taxonomic composition and relative abundance, random sample subsets were generated with the lowest number of reads: 56395 (G-W-1).

The composition of T. matsutake was analyzed first, followed by the composition of the entire fungal community, and finally, a seasonal comparison was conducted (Table 3). As expected, the spot where T. matsutake

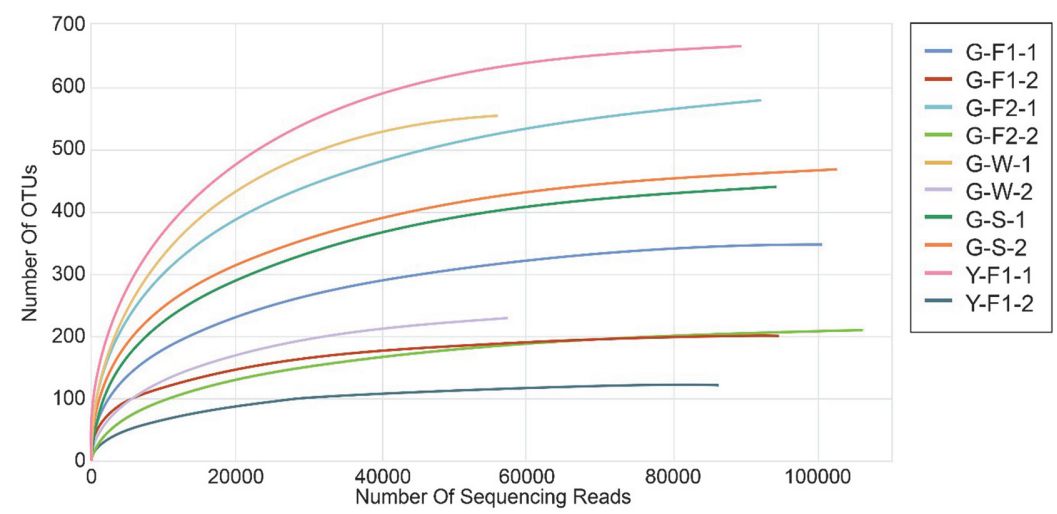

Fig. 1. Rarefaction curves for operational taxonomic units (OTUs) of fungi from all soil samples at Gyeongju and Yeongdeok. OTUs were clustered at $97 \%$ similarity with CD-HIT. This shows the rate of increase in the number of OTUs (species) with the number of acquired sequences. The curves of T. matsutake-dominant soil samples were in low positions. This implies that the fungal diversity is low in T. matsutake-dominant soil samples (X-axis: the number of sequencing reads; Y-axis: the number of OTUs). 
Table 3. Composition (\%) of T. matsutake in each sample (using the sum of reads of Tricholoma matsutake, Tricholoma matsutake_1, and Tricholoma matsutake_7).

\begin{tabular}{cccccccccc}
\hline G-F1-1 & G-F1-2 & G-F2-1 & G-F2-2 & G-W-1 & G-W-2 & G-S-1 & G-S-2 & Y-F1-1 & Y-F1-2 \\
\hline 0.00 & $\mathbf{5 7 . 7 6}$ & 0.12 & $\mathbf{7 9 . 1 3}$ & 0.01 & $\mathbf{7 5 . 1 9}$ & 38.92 & $\mathbf{5 2 . 1 1}$ & 0.00 & $\mathbf{5 2 . 2 5}$ \\
\hline
\end{tabular}

was present was clearly dominated by T. matsutake at more than $51 \%$. The difference between where T. matsutake was present or not present was greater than $50 \%$, except in the G-S samples. This indicates that when the fruiting body of T. matsutake is formed, T. matsutake is definitely a dominant species in that spot. From G-F2-2 to G-W-2 and to G-S-2, the ratio decreased. From G-F2-2 in fall to G-W-2 in winter, the difference was 3.94\%, which was a slight drop. But from G-W-2 to G-S-2, the difference was $23.08 \%$. The difference in G-S where T. matsutake was present or not was $13.19 \%$, which was less than the difference in other samples. In addition, the somewhat low share in the G-S-2 sample, at $52.11 \%$, seems to have naturally decreased as the growth of other fungi became more active with the rise in temperature.

\section{Comparison of Fungal Communities by Region}

First, we conducted an analysis at the phylum level of the taxonomic composition of soil samples from G-F1-1, G-F1-2, G-F2-1, G-F2-2, Y-F1-1, and Y-F1-2. A total of five phyla were identified ( $>1 \%)$ : Basidiomycota, Ascomycota, Mucoromycota, Fungi_p (phylum name unknown), and Mortierellomycota (Fig. 2). Including T. matsutake, Basidiomycota was the dominant phylum in G-F1-1 (48.49\%), G-F1-2 (66.97\%), G-F2-2 (82.84\%), Y-F1-1 (51.42\%), and Y-F1-2 (93.59\%). Ascomycota was dominant only in G-F2-1 (47.64\%). The ratio of Mucoromycota was G-F1-1 (8.22\%), G-F1-2 (14.82\%), G-F2-1 (15.02\%), G-F2-2 (10.65\%), Y-F1-1 (11.63\%), and Y-F1-2 (4.18\%). The ratio of Fungi_p was $6.42 \%$ in G-F2-1 and $0.03 \%$ in G-F2-2. The percentage of Mortierellomycota was as follows: G-F1-1 (2.07\%), G-F1-2 (2.04\%), G-F2-1 (1.11\%), G-F2-2 (0.03\%), Y-F1-1 (1.85\%), and Y-F1-2 (0.05\%). Excluding T. matsutake, Basidiomycota was the dominant phylum in G-F1-1 (48.49\%), Y-F1-1 (51.42\%), and Y-F1-2 (86.57\%), and Ascomycota was dominant in G-F1-2 (36.87\%) and G-F2-1 (47.70\%). To determine the taxonomic composition of fungi except T. matsutake and to analyze the effects of T. matsutake on the composition of other fungi, an additional comparative analysis was performed after excluding the reads of the above analyzed T. matsutake. Excluding T. matsutake, the ratio of Basidiomycota was lower in the regions where T. matsutake grew than in the regions where it did not grow (G-F1-1 > G-F1-2, G-F2-1 > G-F2-2). In the Yeongdeok samples, the ratio was Y-F1-1 < Y-F1-2 because of the unclassified genera Ramaria of Basidiomycota in Y-F1-2, which is mentioned again at the genus level.

At the class level, a total of nine fungal classes found to represent more than $1 \%$ of all the classes were identified: Agaricomycetes, Umbelopsidomycetes, Eurotiomycetes, Leotiomycetes, Dothideomycetes, Sordariomycetes, Mortierellomycetes, Fungi_c (class name was unknown), and GS25 (unnamed). We focused on the classes of the relatively abundant phyla Basidiomycota, Ascomycota, Mucoromycota, and Mortierellomycota. In phylum Basidiomycota, we found Agaricomycetes in G-F1-1 (47.99\%), G-F1-2 (65.95\%), G-F2-1 (26.23\%), G-F2-2 (80.12\%), Y-F1-1 (46.39\%), and Y-F1-2 (93.17\%). Phylum Mucoromycota was represented by Umbelopsidomycetes: G-F1-1 (8.22\%), G-F1-2 (14.82\%), G-F2-1 (14.33\%), G-F2-2 (10.60\%), Y-F1-1 (11.63\%), and Y-F1-2 (4.18\%). Class Umbelopsidomycetes was the second most dominant class in all Sample 2's after class Agaricomycetes, including T. matsutake. For phylum Ascomycota, we found Eurotiomycetes: G-F1-1 (25.05\%), G-F1-2 (2.31\%), G-

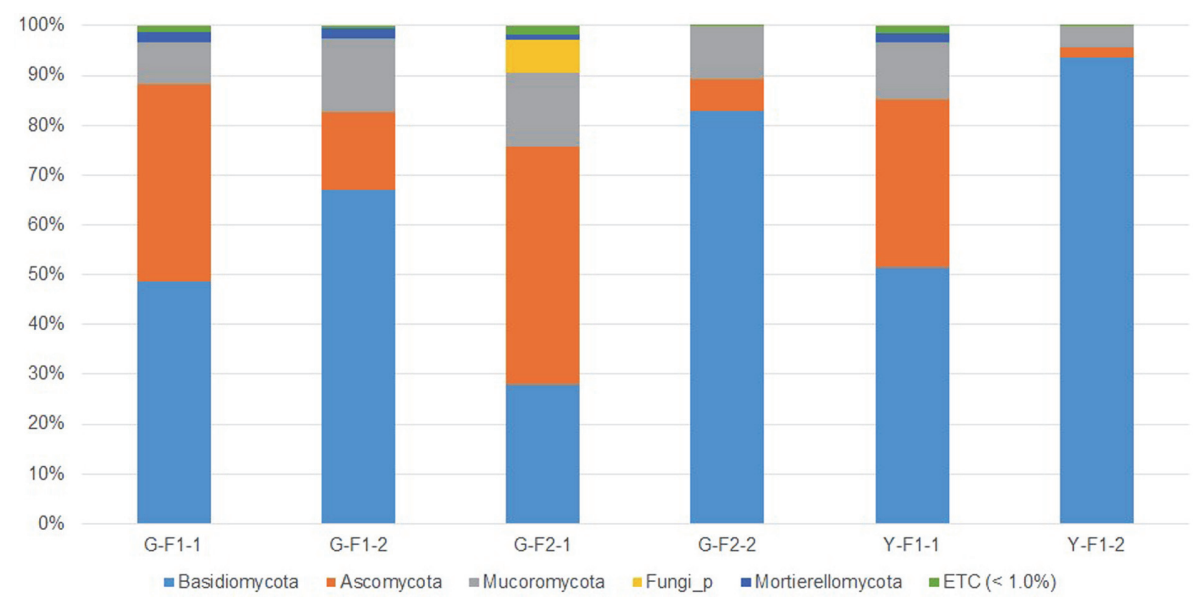

Fig. 2. Taxonomic composition at the phylum level from G-F1-1, G-F1-2, G-F2-1, G-F2-2, Y-F1-1, and Y-F12 samples. Samples of Gyeongju and Yeongdeok collected in autumn were compared. Fungal phyla with a relative abundance greater than $1 \%$ in at least one of the samples are shown and phyla less than $1 \%$ were shown as ETC. Basidiomycota was more abundant in Sample-2 (under fruiting bodies of T. matsutake) than Sample-1 (far from the fairy ring) regardless of regions and was significantly dominant in Y-F1-2 (G-F1: October 2017, G-F2: October 2018, and Y-F1: October 2017). 
Table 4. Gradient heatmap of the taxonomic composition (\%) at the genus level of each sample for comparison by region (including T. matsutake,_uc: unclassified,_g: the genus name was unknown).

\begin{tabular}{lrrrrrr}
\hline Name & G-F1-1 & G-F1-2 & G-F2-1 & G-F2-2 & Y-F1-1 & Y-F1-2 \\
\hline Tricholoma & 5.88 & 58.01 & 0.12 & 79.18 & 0.00 & 52.42 \\
Umbelopsis & 6.77 & 14.50 & 14.29 & 10.60 & 11.47 & 1.68 \\
Ramaria & 0.00 & 0.00 & 0.00 & 0.00 & 0.00 & 38.59 \\
Tylospora & 22.24 & 0.00 & 0.00 & 0.00 & 10.27 & 0.00 \\
Sagenomella & 20.95 & 0.30 & 2.97 & 1.75 & 1.06 & 0.38 \\
Cenococcum & 10.49 & 4.86 & 5.55 & 1.83 & 2.09 & 0.04 \\
Hydnum & 2.47 & 6.39 & 12.49 & 0.00 & 0.00 & 0.00 \\
Oidiodendron & 2.69 & 4.24 & 7.77 & 1.17 & 3.32 & 0.48 \\
Russula & 4.84 & 0.00 & 7.34 & 0.31 & 6.19 & 0.00 \\
Sebacina & 0.88 & 0.00 & 0.00 & 0.00 & 0.11 & 2.96 \\
\hline Penicillium & 1.65 & 0.70 & 2.84 & 0.08 & 0.25 & 0.01 \\
Talaromyces & 0.18 & 0.06 & 0.74 & 0.19 & 0.03 \\
Cladophialophora & 0.02 & 0.93 & 2.02 & 0.10 & 0.16 & 0.14 \\
Phialocephala & 0.23 & 0.32 & 1.26 & 0.01 & 0.51 & 0.49 \\
Fungi_uc_g & 0.34 & 0.05 & 0.37 & 0.00 & 0.45 \\
Herpotrichiellaceae_uc & 0.12 & 0.01 & 0.23 & & Low & 0.00 \\
\hline
\end{tabular}

F2-1 (11.49\%), G-F2-2 (2.32\%), Y-F1-1 (8.51\%), Y-F1-2 (0.56\%); Leotiomycetes: G-F1-1 (3.09\%), G-F1-2 (5.15\%), G-F2-1 (20.92\%), G-F2-2 (2.01\%), Y-F1-1 (14.11\%), and Y-F1-2 (1.22\%); and Dothideomycetes: G-F1-1 (10.70\%), G-F1-2 (5.72\%), G-F2-1 (6.88\%), G-F2-2 (1.84\%), Y-F1-1 (5.82\%), and Y-F1-2 (0.13\%). The classes in phylum Ascomycota had a tendency to show a smaller percentage in Sample 2's than in Sample 1's. For phylum Mortierellomycota, we found Mortierellomycetes: G-F1-1 (2.07\%), G-F1-2 (2.04\%), G-F2-1 (1.11\%), G-F2-2 $(0.03 \%)$, Y-F1-1 (1.85\%), and Y-F1-2 (0.05\%). Mortierellomycetes was present at a lower percentage in Sample 2's than in Sample 1's.

At the genus level, the top 10 most abundant genera were Tricholoma, Umbelopsis, Ramaria, Tylospora, Sagenomella, Cenococcum, Hydnum, Oidiodendron, Russula, and Sebacina (Table 4) in G-F1-1, G-F1-2, G-F2-1, G-F2-2, Y-F1-1, and Y-F1-2. In T. matsutake-dominant G-F1-2 and G-F2-2 samples, Tricholoma, Umbelopsis, Cenococcum, Oidiodendron, Sagenomella, Cladophialophora, Penicillium, Rhizopogon, and Phialocephala were the only genera that existed in common (>0.1\%). In both T. matsutake-minor G-F1-1 and G-F2-1, the genera Sagenomella, Umbelopsis, Cenococcum, Hydnum, Russula, Oidiodendron, Tricholoma, Penicillium, Sistotrema, Phialocephala, Talaromyces, Fungi_uc (unclassified), Herpotrichiellaceae_uc, and Botryosphaeriaceae_uc $(>0.1 \%)$ were present.

Compared to the fungal community from Yeongdeok, Y-F1-1 shared Tylospora with G-F1-1 at 10.27\% and $22.24 \%$, respectively, while none were present in G-F2-1. The genus Tylospora fibrillosa is a nitrogen (N)-tolerant ectomycorrhizal fungus and contributes to the generation of $\mathrm{N}_{2} \mathrm{O}$ [42]. Furthermore, the genus Hydnum in the sample from G-F1-1 and G-F2-1 was not present in Y-F1-1. All species of Hydnum are ectomycorrhizae that coexist with trees such as the Pinaceae and Fagales in Europe [43]. Sebacina (10.90\%), Pseudotomentella (9.60\%), Cadophora (7.61\%), Suillus (4.64\%) were found in Y-F1-1, but in G-F1-1 and G-F2-1, they were either present at very low levels or not present. On the other hand, in Y-F1-1, Umbelopsis (11.43\%), Sagenomella (1.06\%), Cenococcum (2.09\%), Russula (6.19\%), and Oidiodendron (3.32\%) existed in common with G-F1-1 and G-F2-1. Among T. matsutake-dominant samples, G-F1-2, G-F2-2, and Y-F1-2 showed that Tricholoma, Umbelopsis, Oidiodendron, Sagenomella, Cladophialophora and Phialocephala were the only genera that existed in common $(>0.1 \%)$ (Table 4). Most of genus Tricholoma (G-F1-2, 58.01\%; G-F2-2, 79.18\%; Y-F1-2, 52.42\%) was T. matsutake. The genus Umbelopsis was similar but differed by ratio at $14.50 \%, 10.60 \%$, and $1.68 \%$, which was significantly lower only in Y-F1-2. Similarly, the genus Oidiodendron was $4.24 \%$ in G-F1-2, $1.17 \%$ in G-F2-2, but $0.48 \%$ in Y-F12. The genus Sagenomella was $0.30 \%$ and $1.75 \%$, but $0.38 \%$ in Y-F1-2. On the other hand, the genus Ramaria comprised $38.59 \%$ only in Y-F1-2 but was not present in G-F1-2 or G-F2-2. In T. matsutake-minor G-F1-1, G-F21, and Y-F1-1, the genera Umbelopsis, Sagenomella, Russula, Cenococcum, Oidiodendron, Penicillium, Talaromyces, Phialocephala, Herpotrichiellaceae_uc and Fungi_uc $(>0.1 \%)$ were present (Table 4$)$. In particular, the genus Russula was significantly lower in Sample 2, but was 4.84\%, 7.34\%, and 6.19\% in Sample 1.

In our results, Umbelopsis was the most dominant genus, following genus Tricholoma, in most T. matsutakedominant samples. Umbelopsis has been reported on frequently in other studies on the fungal community of T. matsutake. Ogawa and Kawai announced that metabolites produced by Umbelopsis promoted T. matsutake's growth [44]. Also, Umbelopsis was isolated from the P. densiflora rootlet colonized by T. matsutake [45]. A previous study showed that genus Umbelopsis was remarkably more abundant in T. matsutake-dominant soil than in $T$. matsutake-minor soil; in comparison, Mortierella was outstandingly more abundant in T. matsutake-minor soil [25]. Oh and his colleagues reported that some bacteria isolated from the fruiting body of T. matsutake increased 
Table 5. The number of reads for the common species in G-F1-2, G-F2-2, and Y-F1-2 (_uc: unclassified,_s: the species name was unknown).

\begin{tabular}{lccc}
\hline \multicolumn{1}{c}{ Name } & G-F1-2 & G-F2-2 & Y-F1-2 \\
\hline Umbelopsis dimorpha & 13420 & 11133 & 1095 \\
Oidiodendron chlamydosporicum & 450 & 347 & 325 \\
Oidiodendron_uc & 642 & 759 & 26 \\
Sagenomella_diversispora & 34 & 151 & 2 \\
Cenococcum_uc & 4500 & 52 & 6 \\
Sistotrema_uc & 2 & 15 & 10 \\
Cladophialophora_uc & 872 & 7 & 115 \\
Pezicula_uc & 128 & 7 & 85 \\
Oidiodendronpilicola & 657 & 160 & 8 \\
Fungi_uc_s & 47 & 8 & 7 \\
Umbelopsis_uc & 231 & 10 & 379 \\
Sagenomella_uc & 6 & 10 & 24 \\
Tricholoma_uc & 230 & 2 & 125 \\
Basidiomycota_uc_s & 26 & 2 & 3
\end{tabular}

the growth of Umbelopsis, suggesting a strong link between T. matsutake and Umbelopsis [46]. Umbelopsis quickly grows into a nutrient-rich environment of fresh litters in the fall, when T. matsutake fruiting body is produced. Umbelopsis is also known as one of the common fungi associated with seedlings and healthy roots of pine tree [47, 48]. Tominaga discovered the relationship between T. matsutake and Umbelopsis by separating Umbelopsis from both mycorrhizae and the fruiting body of T. matsutake [49].

However, in the T. matsutake-minor G-F2-1 and Y-F1-1 samples, Umbelopsis was the most dominant genus at $14.29 \%$ and $11.47 \%$ each, respectively. Ogawa discovered that the zone of shiro at which fruiting body of T. matsutake forms Mortierella sp. (probably Umbelopsis) increased, and at the end of the shiro, Mortierella sp. and the other root fungi were commonly abundant [50]. Thus, we suggested that the sampling spots of G-F2-1 and YF1-1 could be near the end of the shiro and Umbelopsis was abundant in that spot. The interaction between Umbelopsis and T. matsutake remains uncertain and thus further study is needed to determine which mechanisms are interacting with them.

On the other hand, in our samples, there were several genera that were lower in Sample 2 than in Sample 1. The ratios of these genera were as follows: Sagenomella (G-F1-1, 20.95\% and G-F1-2, 0.30\%; G-F2-1, 2.97\% and G-F22, 1.75\%; Y-F1-1, 1.06\% and Y-F1-2, 0.38\%), Cenococcum (10.49\% and 4.86\%; 5.55\% and $1.83 \% ; 2.09 \%$ and $0.04 \%)$, Russula (4.84\% and $0.00 \% ; 7.34 \%$ and $0.31 \% ; 6.19 \%$ and $0.00 \%)$, and Penicillium $(1.65 \%$ and $0.70 \% ; 2.84 \%$ and $0.11 \% ; 2.96 \%$ and $0.03 \%)$.

For comparison of species comprising less than $1 \%$ of the community, only this number was compared with samples before normalization. Vaario and colleagues found that Tomentellopsis sp., Cortinarius biformis, Tylospora sp., tomentellopsis submollis, and Trichoderma viride were positively correlated with the presence of T. matsutake in soil above the fairy ring of T. matsutake [14]. Additionally, species Piloderma sp. 4, Clavulina cf. amethystine, and Piloderma sp. 3 were positively correlated with the presence of T. matsutake in soil in the fairy ring of T. matsutake. However, in our analysis, each of these species were present at a significantly low level $(<0.1 \%)$, and they existed in both T. matsutake-dominant and -minor soil. Thus, it is hard to say that they had a positive relationship. Furthermore, Tylospora_uc (unclassified into sublevel) comprised $22.24 \%$ of G-F1-1 and $10.27 \%$ of Y-F-1 but $0.00 \%$ in all T. matsutake-dominant samples, which suggests that they were not in a positive relationship. In comparison with the number of reads, there was only one species, Aspergillus cervinus, which was present in G-F1-2 (6 reads) and G-F2-2 (7 reads) but not in G-F1-1 and G-F2-1. Aspergillus cervinus was originally isolated from African soil [51] and generates the quinol terremutin and 3,6-dihydroxy-2,5-toluquinone [52, 53]. There were no species present in G-F1-2, G-F2-2, and Y-F1-2 that were not present in G-F1-1, G-F2-1, and Y-F21. However, in G-F1-2, G-F2-2, and Y-F1-2, there were 14 species in common with their number of reads in Table 5.

\section{Comparison of Fungal Communities by Season}

A total of seven distinct fungal phyla were found to represent more than $1 \%$ of the total phyla in the G-F2, G-W, and G-S samples (Fig. 3). From G-F2-2 to G-W-2 to G-S-2, the ratio of the phylum Basidiomycota gradually dropped from $82.84 \%$ to $77.01 \%$ to $60.28 \%$, respectively. Additionally, the phylum Mucoromycota was reduced from $10.65 \%$ to $7.29 \%$ to $5.17 \%$. In contrast, the level of phylum Ascomycota was increasingly higher at $6.37 \%$, $14.70 \%$, and $27.29 \%$, respectively. Likewise, other phyla showed a small difference in ratio, including Fungi_p $(0.03 \%, 0.37 \%$, and $4.30 \%)$, Mortierellomycota $(0.03 \%, 0.14 \%$, and $1.72 \%)$, Rozellomycota $(0.02 \%, 0.21 \%$, and $0.52 \%)$, and Chytridiomycota $(0.01 \%, 0.09 \%$, and $0.17 \%)$.

A total of 11 fungal classes representing more than $1 \%$ of all the classes in each sample were identified: Agaricomycetes, Leotiomycetes, Eurotiomycetes, Umbelopsidomycetes, Dothideomycetes, Sordariomycetes, Fungi_c, GS25, Mortierellomycetes, Lecanoromycetes, and Rozellomycotina cls Incertae sedis (unknown). We focused on the classes of the relatively abundant phyla Basidiomycota, Ascomycota, Mucoromycota, and Mortierellomycota. For phylum Basidiomycota, Agaricomycetes were present as follows: G-F2-2, 80.12\%; G-W-2, $76.18 \%$; and G-S-2, 59.15\%. From G-F2-2 to G-W-2 to G-S-2, the ratio of Agaricomycetes decreased. For phylum 


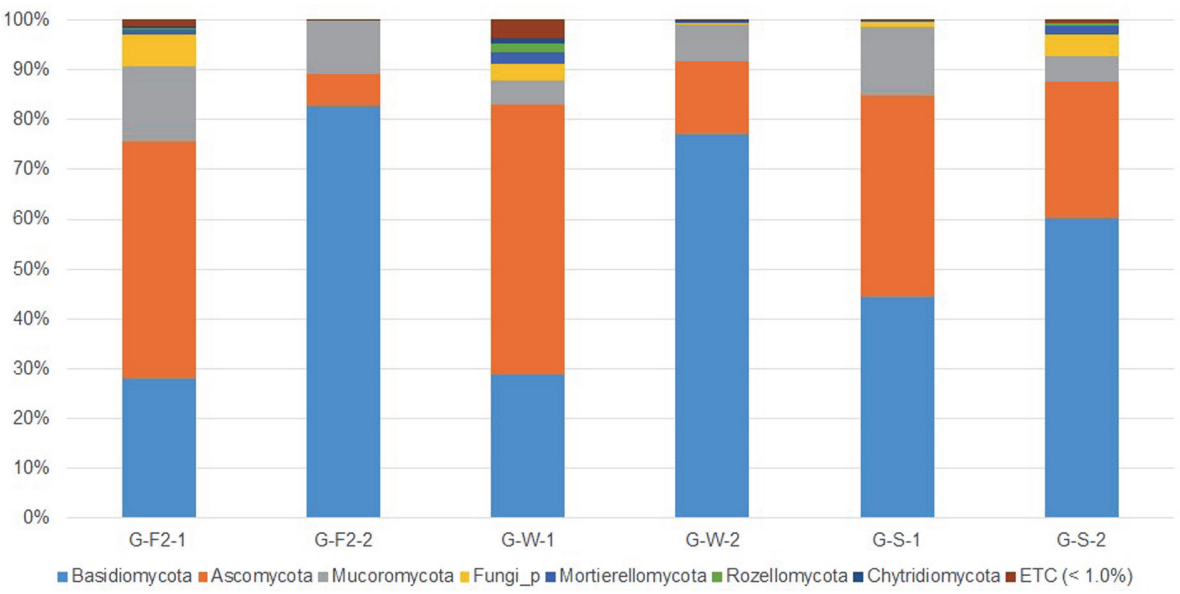

Fig. 3. Taxonomic composition at the phylum level from the G-F2-1, G-F2-2, G-W-1, G-W-2, G-S-1, G-S-2 samples. Samples of only Gyeongju collected in autumn, winter, spring were compared. Fungal phyla with a relative abundance greater than $1 \%$ in at least one of the samples are shown and phyla less than $1 \%$ were shown as ETC. From G-F2-2 to G-W-2 to G-S-2, the ratio of the phylum Basidiomycota gradually dropped (G-F2: October 2018, G-W: February 2019, and GS: June 2019, -1: far from the fairy ring, -2: under fruiting bodies of T. matsutake).

Ascomycota, the ratios were as follows, respectively: Leotiomycetes: $2.01 \%, 3.57 \%$, and $10.81 \%$, Eurotiomycetes: $2.32 \%, 9.14 \%$, and $11.31 \%$; Dothideomycetes: $1.84 \%, 1.23 \%$, and $1.12 \%$; Sordariomycetes: $0.08 \%, 0.58 \%$, and $3.45 \%$; Lecanoromycetes: $0.01 \%, 0.02 \%$, and $0.03 \%$. From G-F2-2 to G-W-2 to G-S-2, Leotiomycetes, Eurotiomycetes, Sordariomycetes, and Lecanoromycetes increased; in contrast, Dothideomycetes decreased. For phylum Mucoromycota, we found Umbelopsidomycetes: 10.60\%, 7.23\%, and 4.88\%. From G-F2-2 to G-W-2 to G$\mathrm{S}-2$, the proportion of this class decreased. For phylum Mortierellomycota, we found Mortierellomycetes at $0.03 \%$, $0.14 \%$, and $1.72 \%$, and the percentages were increasing.

At the genus level, Oidiodendron of Leotiomycetes increased from $1.17 \%$ to $2.21 \%$ to $3.25 \%$ in G-F2-2, G-W-2, and G-S-2, respectively. Likewise, the genus Hyaloscyphaceae_g (genus name unknown) of Leotiomycetes increased from $0.42 \%$ to $0.58 \%$ to $5.61 \%$. In contrast, in the case of genus Umbelopsis, the proportion was reduced in the order of $10.60 \%$ to $7.19 \%$ to $4.81 \%$. It was also the second largest proportion of genus in each sample after T. matsutake, which may be associated with a gradual decrease of $79.01 \%$ to $75.22 \%$ to $51.98 \%$. As mentioned above, Umbelopsis was not a significantly enriched genus in T. matsutake-dominant samples, but we suggest that it is positively related to T. matsutake. In addition, the genus Cenococcum decreased from $1.83 \%$ to $1.14 \%$ to $0.83 \%$ as the seasons changed.

Park and his colleagues conducted in vitro transplantation of $P$. densiflora seedlings inoculated with T. matsutake and showed the different results from our study covering several points [54]. Our study did not have a significant change of the transplantation which they conducted, but only observed seasonal changes and regional differences in the wild environment in which T. matsutake grows, so there was a clear difference from the in vitro experiment. Fusarium was also found very minimal in our sample and F. oxysporum was never found. This difference may result from the large environmental difference between the artificially established greenhouse and soils in which T. matsutake and its associated fungi have already naturally established their community. They showed as the number of T. matsutake decreases, Suillus increases. Very little Suillus existed in our Sample-1 and we share the same opinion that Suillus and T. matsutake probably have a negative relationship though the reason is unclear. Cylindrocarpon pauciseptatum showed a significantly negative correlation with T. matsutake in Park's work and was also not found in our samples. We assume that it is because of a feature of our sampling area or a wild soil condition of T. matsutake and other fungi communities that were formed long ago. The exact reason is not clear, but the difference from the in vitro experiment was evident, and this interesting relationship between these fungi and T. matsutake requires further research.

T. matsutake is a mushroom that can be found only in autumn when the temperature has cooled. Annual production of T. matsutake is determined by the rainfall and temperature in fall [55]. Compared to Gyeongju, the sampling sites at Yeongdeok are affected by the area's proximity to the ocean. The average daily temperature on the day the sample was collected in Gyeongju was $19.9^{\circ} \mathrm{C}$ and in Yeongdeok it was $11.5^{\circ} \mathrm{C}$ [56]. At the time of sampling, the low read number of Yeongdeok samples seems to have been affected by the temperature. The average temperature of October 2018 was $13.7^{\circ} \mathrm{C}$, lower than in October $2017\left(15.6^{\circ} \mathrm{C}\right)$. The precipitation in October 2018 was $223.2 \mathrm{~mm}$ per month, a significant increase from October 2017 (49.7 mm per month). The production of T. matsutake fruiting body increased rapidly in 2018 due to high rainfall in South Korea $[7,56]$. This can be the reason why the ratio of T. matsutake in G-F2-2 is higher than in G-F1-2. According to studies by Chung and colleagues, the rate of mycelium occurrence was high in areas with high soil moisture content and this supports the above results [57]. Chung also found that the iron ion content in T. matsutake soil was higher than in soil where T. matsutake was not dominant. Vaario and his colleagues, however, said that T. matsutake did not appear to be 
related to a specific soil chemistry [14]. On the other hand, $\mathrm{pH}$ around the area where T. matsutake grows was relatively acidic $[14,57,58]$. However, soil of more than $\mathrm{pH} 5.0$ was also found [59]. Thus, the correlation between T. matsutake, the fungal community, and soil chemistry needs to be further studied. Also, soil chemistry and its seasonal changes related to T. matsutake need to be measured in further studies in Gyeongju and Yeongdeok soil.

In this study we analyzed the fungal community in the soil under fruiting bodies of T. matsutake, and also where the fairy ring of T. matsutake was no longer present. We compared it with the fungal community in soil from other regions. In addition, the same spot was investigated three times at intervals of four months at the same spot from fall to the following spring, to observe changes in the soil fungal community related to T. matsutake.

We got a high enough number of valid reads in each sample to analyze the fungal community. The valid reads of G-F2-2 were the highest, at 106,048, and those of G-W-1 were the fewest, at 56,395. The OTUs of most samples under fruiting bodies of T. matsutake were lower than OTUs from samples where T. matsutake was no longer present. This indicates that the fungal diversity is low in soil where T. matsutake is dominant. Therefore, the mycelia of T. matsutake appear to repress the growth of other fungi and are dominant in the soil fungal community. The soil under T. matsutake fruiting bodies was clearly dominated by T. matsutake at more than $51 \%$. The difference between where T. matsutake was present or not was more than 50\%, except in G-S-1 and G-S-2. This implies that when the fruiting body of T. matsutake is formed, T. matsutake is a dominant species in that spot. In the samples from Yeongdeok, the number of valid reads was slightly lower than that from Gyeongju.

Basidiomycota was the dominant phylum in most samples. The classes in phylum Ascomycota had a tendency to show a smaller percentage in Sample 2 than in Sample 1. T. matsutake-dominant G-F1-2 and G-F2-2 samples had only the following genera in common: Tricholoma, Umbelopsis, Cenococcum, Oidiodendron, Sagenomella, Cladophialophora, Penicillium, Rhizopogon, and Phialocephala. In both T. matsutake-minor G-F1-1 and G-F2-1, the genera Sagenomella, Umbelopsis, Cenococcum, Hydnum, Russula, Oidiodendron, Tricholoma, Penicillium, Sistotrema, Phialocephala, Talaromyces, Fungi_uc, Herpotrichiellaceae_uc, and Botryosphaeriaceae_uc were present. In T. matsutake-dominant samples, G-F1-2, G-F2-2, and Y-F1-2 had only the genera Tricholoma, Umbelopsis, Oidiodendron, Sagenomella, Cladophialophora, and Phialocephala in common. In T. matsutakeminor G-F1-1, G-F2-1, and Y-F1-1, the genera Umbelopsis, Sagenomella, Russula, Cenococcum, Oidiodendron, Penicillium, Talaromyces, Phialocephala, Herpotrichiellaceae_uc and Fungi_uc were present.

With fewer reads from winter samples, the overall number was reduced, but in the spring, the number of samples and reads increased again. From fall to the following spring, the ratio of phyla Basidiomycota and Mucoromycota gradually dropped. In contrast, the phylum Ascomycota increased. A total of 11 fungal classes were found to represent more than $1 \%$ of the total classes in each sample. The classes Leotiomycetes, Eurotiomycetes, Sordariomycetes, Lecanoromycetes, and Mortierellomycetes increased, while Dothideomycetes and Umbelopsidomycetes decreased. The genus Umbelopsis accounted for the second-largest proportion of each sample after T. matsutake. We suggest that it is positively related to T. matsutake.

This study provides a foundation for understanding the ecological relationships between T. matsutake and other fungi in soil. However, further studies are necessary to analyze and investigate how T. matsutake interacts with other fungi, microorganisms, and roots of $P$. densiflora. Although several authors have published results from similar research on the T. matsutake-related microbial community, it was difficult to find commonality because of sampling methods and regional differences. Therefore, it is necessary to find a method that is accurate, uniform, and standardized to analyze the T. matsutake-related microbial community.

\section{Acknowledgments}

This study was supported by the Basic Science Research Program through the National Research Foundation of Korea (NRF), Ministry of Education (2016R1A6A1A05011910), and the Research Institute for Dok-do and Ulleung-do Island of Kyungpook National University.

\section{Conflict of Interest}

The authors have no financial conflicts of interest to declare.

\section{References}

1. Ahn JS, Lee KH. 1986. Studies on the volatile aroma components of edible mushroom (Tricholoma matsutake) of Korea. J. Kor. Soc. Food Nutr. 15: 253-257.

2. Hoshi H, Yagi Y, Iijima H, Matsunaga K, Ishihara Y, Yasuhara T. 2005. Isolation and characterization of a novel immunomodulatory alpha-glucan-protein complex from the mycelium of Tricholoma matsutake in basidiomycetes. J. Agric. Food Chem. 53: 8948-8956.

3. Kim JY, Byeon SE, Lee YG, Lee JY, Park J, Hon, EK, et al. 2008. Immunostimulatory activities of polysaccharides from liquid culture of pine-mushroom Tricholoma matsutake. J. Microbiol. Biotechnol. 18: 95-103.

4. Kim SY, Go KC, Song YS, Jeong YS, Kim EJ, Kim, BJ. 2014. Extract of the mycelium of T. matsutake inhibits elastase activity and TPAinduced MMP-1 expression in human fibroblasts. Int. J. Mol. Med. 34: 1613-1621.

5. Hou Y, Ding X, Hou W, Zhong J, Zhu H, Ma B, et al. 2013. Anti-microorganism, anti-tumor, and immune activities of a novel polysaccharide isolated from Tricholoma matsutake. Pharmacogn Mag. 9: 244-249.

6. Ohnuma N, Amemiya K, Kakuda R, Yaoita Y, Machida K, Kikuchi M. 2000. Sterol constituents from the edible mushrooms, Lentinula edodes and Tricholoma matsutake. Chem. Pharm. Bull. 48: 749-751.

7. Park C. 2020. 2019 Production of Forest Products. Korea Forest Service.

8. Yamada A, Kanekawa S, Ohmasa M. 1999. Ectomycorrhiza formation of Tricholoma matsutake on Pinus densiflora. Mycoscience 40: 193-198.

9. Yamada A, Maeda K, Ohmasa M. 1999. Ectomycorrhiza formation of Tricholoma matsutake isolates on seedlings of Pinus densiflora in vitro. Mycoscience 40: 455-463. 
10. Narimatsu M, Koiwa T, Masaki T, Sakamoto Y, Ohmori H. 2015. Relationship between climate, expansion rate, and fruiting in fairy rings ('shiro') of an ectomycorrhizal fungus Tricholoma matsutake in a Pinus densiflora forest. Fungal Ecol. 15: 18-28.

11. Kataoka R, Siddiqui ZA, Kikuchi J, Ando M, Sriwati R, Nozaki A, et al. 2012. Detecting nonculturable bacteria in the active mycorrhizal zone of the pine mushroom Tricholoma matsutake. J. Microbiol. 50: 199-206.

12. Kim M, Yoon H, You YH, Kim YE, Woo JR. 2013. Metagenomic analysis of fungal communities inhabiting the fairy ring zone of Tricholoma matsutake. J. Microbiol. Biotechnol. 23: 1347-1356.

13. Lian C, Narimatsu M, Nara K, Hogetsu T. 2006. Tricholoma matsutake in a natural Pinus densiflora forest: correspondence between above- and below-ground genets, association with multiple host trees and alteration of existing ectomycorrhizal communities. New Phytol. 171: 825-836.

14. Vaario LM, Fritze H, Spetz P, Heinonsalo J, Hanajík P, Pennanen T. 2011. Tricholoma matsutake dominates diverse microbial communities in different forest soils. Appl. Environ. Microbiol. 77: 8523-8531.

15. Corrales A, Arnold AE, Ferrer A, Turner BL, Dalling JW. 2016. Variation in ectomycorrhizal fungal communities associated with Oreomunnea mexicana (Juglandaceae) in a Neotropical montane forest. Mycorrhiza 26: 1-17.

16. Kirk PM, Cannon PF, David JC, Stalpers JA. 2001. Ainsworth and Bisby's Dictionary of the Fungi, 9th Ed. CABI publishing, Wallingford. Uk.

17. Peterson RL, Massicotte HB, Melville LH. 2004. Mycorrhizas: anatomy and cell biology, National Research Council Research Press, Ottawa. USA.

18. Roy M, Schimann H, Braga-Neto R, Da Silva RAE, Duque J, Frame D, et al. 2016. Diversity and distribution of ectomycorrhizal fungi from Amazonian lowland white-sand forests in Brazil and French Guiana. Biotropica 48: 90-100.

19. Wang B, Qiu YL. 2006. Phylogenetic distribution and evolution of mycorrhizas in land plants. Mycorrhiza 16: 299-363.

20. Agerer R. 2006. Fungal relationships and structural identity of their ectomycorrhizae. Mycol. Prog. 5: 67-107.

21. Harley JL. 1989. The significance of mycorrhiza. Mycol. Res. 92: 129-139.

22. Lang C, Seven J, Polle A. 2011. Host preferences and differential contributions of deciduous tree species shape mycorrhizal species richness in a mixed central European forest. Mycorrhiza 21: 297-308.

23. Sabella E, Nutricati E, Aprile A, Miceli A, Sorce C, Lorenzi R, et al. 2015. Arthrinium phaeospermum isolated from Tuber borchii ascomata: the first evidence for a "Mycorrhization Helper Fungus"? Mycol. Progress 14: 59.

24. Gottel NR, Castro HF, Kerley M, Yang Z, Pelletier DA. 2011. Distinct microbial communities within the endosphere and rhizosphere of Populus deltoides roots across contrasting soil types. Appl. Environ. Microbiol. 77: 5934-5944.

25. Oh SY, Fong JJ, Park MS, Lim YW. 2016. Distinctive feature of microbial communities and bacterial functional profiles in Tricholoma matsutake dominant soil. PLoS One 11: e0168573.

26. Zak DR, Holmes WE, White DC, Peacock AD, Tilman D. 2003. Plant diversity, soil microbial communities, and ecosystem function: are there any links? Ecology 84: 2042-2050.

27. Hollister EB, Schadt CW, Palumbo AV, Ansley RJ, Boutton TW. 2010. Structural and functional diversity of soil bacterial and fungal communities following woody plant encroachment in the southern Great Plains. Soil Biol. Biochem. 42: 1816-1824.

28. Kernaghan G. 2005. Mycorrhizal diversity: Cause and effect? Pedobiologia 49: 511-520.

29. Peay KG, Baraloto C, Fine PV. 2013. Strong coupling of plant and fungal community structure across western Amazonian rainforests. ISME J. 7: 1852-1861.

30. Wu YT, Wubet T, Trogisch S, Both S, Scholten T. 2013. Forest age and plant species composition determine the soil fungal community composition in a Chinese subtropical forest. PLoS One 8: e66829.

31. Baptista P, Reis F, Pereira E, Tavares RM, Santos PM, Richard F, et al. 2015. Soil DNA pyrosequencing and fruitbody surveys reveal contrasting diversity for various fungal ecological guilds in chestnut orchards. Environ. Microbiol. Rep. 7: 946-954.

32. Handelsman J. 2004. Metagenomics: application of genomics to uncultured microorganisms. Microbiol. Mol. Biol. Rev. 68: 669-685.

33. Shokralla S, Spall JL, Gibson JF, Hajibabaei M. 2012. Next-generation sequencing technologies for environmental DNA research. Mol. Ecol. 21: 1794-1805.

34. Streit WR, Schmitz RA. 2004. Metagenomics--the key to the uncultured microbes. Curr. Opin. Microbiol. 7: 492-498.

35. Cannon PF, Kirk PM. 2007. Fungal Families of the World, Wallingford, UK: CABI.

36. Panaro NJ, Yuen PK, Sakazume T, Fortina P, Kricka LJ. 2000. Evaluation of DNA fragment sizing and quantification by the agilent 2100 bioanalyzer. Clin. Chem. 46: 1851-1853.

37. Caporaso JG, Lauber CL, Walters WA, Berg-Lyons D, Huntley J, Fierer N, et al. 2012. Ultra-high-throughput microbial community analysis on the Illumina HiSeq and MiSeq platforms. ISME J. 6: 1621-1624.

38. Bolger AM, Lohse M, Usadel B. 2014. Trimmomatic: a flexible trimmer for Illumina sequence data. Bioinformatics 30: 2114-2120.

39. Li W, Godzik A. 2006. Cd-hit: a fast program for clustering and comparing large sets of protein or nucleotide sequences. Bioinformatics 22: 1658-1659.

40. Heck KL, Van Belle G, Simberloff D. 1975. Explicit calculation of the rarefaction diversity measurement and the determination of sufficient sample size. Ecology 56: 1459-1461.

41. Schloss PD, Westcott SL, Ryabin T, Hall JR, Hartmann M. 2009. Introducing mothur: open-source, platform-independent, community-supported software for describing and comparing microbial communities. Appl. Environ. Microbiol. 75: 7537-7541

42. Prendergast-Miller MT, Baggs EM, Johnson D. 2011. Nitrous oxide production by the ectomycorrhizal fungi Paxillus involutus and Tylospora fibrillosa. FEMS Microbiol. Lett. 316: 31-35.

43. Niskanen T, Liimatainen K, Nuytinck J, Kirk P, Ibarguren IO, Garibay-Orijel R, et al. 2018. Identifying and naming the currently known diversity of the genus Hydnum, with an emphasis on European and North American taxa. Mycologia 110: 890-918.

44. Ogawa M. 1976. Studies on the artificial reproduction of Tricholoma matsutake (S. Ito et Imai) Sing. III. Effects of growth promotion of natural products on the vegetative growth of T. matsutake. Trans Mycol. Soc. Jpn. 17: 492-498.

45. You YH, Yoon HJ, Woo JR, Rim SO, Lee JH, Kong WS, et al. 2011. Diversity of endophytic fungi isolated from the rootlet of Pinus densiflora colonized by Tricholoma matsutake. Kor. J. Mycol. 39: 223-226.

46. Oh SY, Kim M, Eimes JA, Lim YW. 2018. Effect of fruiting body bacteria on the growth of Tricholoma matsutake and its related molds. PLoS One 13: e0190948.

47. Osono T. 2006. Role of phyllosphere fungi of forest trees in the development of decomposer fungal communities and decomposition processes of leaf litter. Can. J. Microbiol. 52: 701-716.

48. Summerbell RC. 2005. From Lamarckian fertilizers to fungal castles: recapturing the pre-1985 literature on endophytic and saprotrophic fungi associated with ectomycorrhizal root systems. Stud. Mycol. 53: 191-256.

49. Tominaga Y. 1963. Studies on the life history of Japanese pine mushroom, Armillaria matsutake Ito et Imai. Bull. Hiroshima Agric. College 2: 105-145.

50. Ogawa M. 1977. Microbial ecology of mycorrhizal fungus Tricholoma matsutake (S. Ito et Imai) Sing. in pine forest. III. Fungal florae in shiro soil and on the mycorrhiza. Bull. Government Forest Experimental Station 293: 105-170.

51. Massee GE. 1914. Aspergillus cervinus Massee. Kew Misc. Bull. 4: 158.

52. Elsohly HN, Slatkin DJ, Schiff Jr PL, Knapp JE. 1974. Metabolites of Aspergillus cervinus massee (Moniliaceae). J. Pharm. Sci. 63: $1632-1633$ 
53. Chen AJ, Varga J, Frisvad JC, Jiang XZ, Samson RA. 2016. Polyphasic taxonomy of Aspergillus section Cervini. Stud. Mycol. 85: 65-89. 54. Park KH, Oh SY, Yoo S, Park MS, Fong JJ, Lim YW. 2020. Successional change of the fungal microbiome pine seedling roots inoculated with Tricholoma matsutake. Front. Microbial. 11: 2413.

55. Cho DH, Lee KJ. 1995. A relationship between climatic factors and matsutake productions in 29 sites during a 10 -year period in Korea. J. Korean Soc. For. Sci. 84: 277-285.

56. Korea Meteorological Administration. 2015. Climate statistical analysis. Available from https://data.kma.go.kr/cmmn/main.do. Accessed Mar. 22, 2021

57. Chung DY, Lee KS, Lee JS, Youn YN. 2008. Characteristics of a forest soil on pine mushroom habitat located in Ponghwa, Kyungbuk and Gansung, Kangwon. 1. Physical and chemical properties of O horizon and surface soil. Kor. J. Soil Sci. Fert. 41: 206-213.

58. Kim M, Yoon H, Kim YE, Kim YJ, Kong WS, Kim JG. 2014. Comparative analysis of bacterial diversity and communities inhabiting the fairy ring of Tricholoma matsutake by barcoded pyrosequencing. I. Appl. Microbiol. 117: 699-710.

59. Li Q, Li X, Chen C, Li S, Huang W, Xiong C, et al. 2016. Analysis of bacterial diversity and communities associated with Tricholoma matsutake fruiting bodies by barcoded pyrosequencing in Sichuan province, southwest China. J. Microbiol. Biotechnol. 26: 89-98. 